\title{
Behavioral and physiological indicators of stress coping styles in larval zebrafish
}

\section{Christian Tudorache, Anique ter Braake, Mara Tromp, Hans Slabbekoorn \& Marcel J. M. Schaaf}

To cite this article: Christian Tudorache, Anique ter Braake, Mara Tromp, Hans Slabbekoorn \& Marcel J. M. Schaaf (2015) Behavioral and physiological indicators of stress coping styles in larval zebrafish, Stress, 18:1, 121-128, DOI: 10.3109/10253890.2014.989205

To link to this article: http://dx.doi.org/10.3109/10253890.2014.989205

Accepted author version posted online: 19

Nov 2014.

Published online: 08 Jan 2015.

Submit your article to this journal $₫$

Џلll Article views: 284

Q View related articles ¿ك

View Crossmark data $־$

Citing articles: 7 View citing articles ¿ 


\title{
Behavioral and physiological indicators of stress coping styles in larval zebrafish
}

\author{
Christian Tudorache, Anique ter Braake, Mara Tromp, Hans Slabbekoorn, and Marcel J. M. Schaaf \\ Institute of Biology, Leiden University, Leiden, The Netherlands
}

\begin{abstract}
Different individuals cope with stressors in different ways. Stress coping styles are defined as a coherent set of individual behavioral and physiological differences in the response to a stressor which remain consistent across time and context. In the present study, we have investigated coping styles in larval zebrafish (Danio rerio) at 8 days post-fertilization. Larvae were separated into two groups, according to the emergence sequence from a darkened into a novel well-lit environment, early (EE) and late (LE) emergers. We used brief periods of netting as a stressor. Swimming behavior and kinematics before and after netting stress were analyzed, as were whole-body cortisol levels before and at 10,30 and $60 \mathrm{~min}$ after the stress event. The results show that general swimming activity was different between EE and LE larvae, with lower baseline cumulative distance and more erratic swimming movements in EE than in LE larvae. EE larvae showed a faster recovery to baseline levels after stress than LE larvae. Cortisol baseline levels were not different between EE and LE larvae, but peak levels after stress were higher and the recovery towards basal levels was faster in EE than in LE larvae. This study shows that coping styles are manifest in zebrafish larvae, and that behavior and swimming kinematics are associated with different cortisol responses to stress. A better understanding of the expression of coping styles may be of great value for medical applications, animal welfare issues and conservation.
\end{abstract}

Keywords

Behavioral syndrome, cortisol, HPA axis, kinematics, personality, physiology, stress

\section{History}

Received 25 August 2014

Revised 3 November 2014

Accepted 4 November 2014

Published online 8 January 2015

\section{Introduction}

Stress coping styles are individual sets of behavioral and physiological traits that are consistent across time and context (Conrad et al., 2011; Koolhaas et al., 1999; Øverli et al., 2007). Behavioral responses can vary individually along a proactive-reactive continuum. This may affect survival and reproductive success and has been studied in a variety of species, including fish (e.g. Coleman \& Wilson, 1998; Huntingford, 1976; Raoult et al., 2012; Schjolden et al., 2005). Physiological responses can also vary individually, for example expressed in the stress-induced secretion of cortisol, arising from activation of the hypothalamus-pituitaryinterrenal (HPI) axis in fish (Wendelaar-Bonga, 1997). The cortisol response depends on the intensity, duration, controllability and predictability of the stressor (De Kloet et al., 1998; Korte et al., 2005) and chronically elevated cortisol levels can have deleterious effects (e.g. Bernier et al., 2004; Consten et al., 2002; Schreck et al., 2001).

Zebrafish (Danio rerio) have become an important model organism for neuro-pharmacological and behavioral research on anxiety and stress (for a review see

Correspondence: Christian Tudorache, Institute of Biology, Leiden University, Sylviusweg 72, 2333 BE Leiden, The Netherlands. Tel: +31 071527 5027. E-mail: c.tudorache@ biology.leidenuniv.nl
Steenbergen et al., 2011). Earlier studies in this species have for example shown a clear correlation between an external stressor and the rise and recovery pattern in cortisol levels (Ramsay et al., 2006), as well as between anxiety-related behaviors and cortisol (Egan et al., 2009). Oswald et al. (2012) investigated shy and bold selection lines and although they found variation in cortisol level to be related to stressor and sex but not to behavioral type, they revealed divergent gene expression patterns with an up-regulation of glucocorticoid receptor activity for the shy line. Furthermore, we recently revealed that different behavioral responses of non-selected wild-type zebrafish correlate with different cortisol recovery profiles after an external stressor (Tudorache et al., 2013).

Coping styles can already be present at a larval stage, as is known from amphibians and fish (e.g. Höglund et al., 2008; Koprivnikar et al., 2012). At this stage they may determine decisions critical for survival to a reproductive stage (e.g. Andersson et al., 2013; McCormick \& Meekan, 2010). Zebrafish are also very suitable to study external stressors and consistent behavioral strategies in larvae (e.g. Budaev \& Andrew, 2009; Champagne et al., 2010; Vignet et al., 2013) as they already have a fully functional HPI axis from $97 \mathrm{~h}$ post-fertilization (Alsop \& Vijayan, 2008). However, it is unknown yet whether the correlation between behavioral type and stressor-induced cortisol recovery profile in larvae is as tight as in adult zebrafish (Tudorache et al., 2013). 
The objective of the current study was to investigate the association between behavioral and physiological indicators of coping style in zebrafish larvae. We first explored the validity of splitting up groups in behavioral types based on the ranking of individual emergence time in a standardized test. Subsequently, we investigated early and late emerging individuals (EE and LE) by assessing swimming kinematics and cortisol levels before and after a stress event.

\section{Materials and methods}

\section{Zebrafish maintenance and production of larvae}

Zebrafish (D. rerio, ABTL) were raised in 101 tanks at $28^{\circ} \mathrm{C}$ in a $12-12 \mathrm{~h}$ light-dark cycle $(8 \mathrm{am}-8 \mathrm{pm})$. Fertilization was performed by natural spawning (family crossings) at the beginning of the light period. Eggs were collected, transferred to Petri dishes containing egg water $(60 \mu \mathrm{g} / \mathrm{ml}$ " Instant Ocean' sea salts) and placed on a white background at $28^{\circ} \mathrm{C}$ (100 eggs/dish). At 5 days post-fertilization (dpf), larvae were transferred to the experimental room $\left(24^{\circ} \mathrm{C}\right)$. Experimental tests were performed at $8 \mathrm{dpf}$. For an overview of the experimental timeline see Figure 1.

\section{Emergence test}

The emergence test concerned a group splitting procedure based on the emergence sequence from a darkened holding compartment through a narrow outlet into a well-lit compartment, dividing larvae into three groups of equal numbers and retaining the two groups of extreme behavioral phenotypes (early emerging, EE and late emerging, LE) for further study, similar to the test for adult zebrafish described in Tudorache et al. (2013). The experimental setup consisted of a circular darkened holding compartment (Petri dish $3.3 \mathrm{~cm}$ diameter $\times 1 \mathrm{~cm}$ height $)$, connected by a passage tube $(0.5 \mathrm{~cm}$ diameter $\times 0.6 \mathrm{~cm}$ length) to a lit circular novel environment compartment $(3.3 \mathrm{~cm}$ diameter $\times 1 \mathrm{~cm}$ height $)$. The emergence test was conducted every day before $10 \mathrm{am}$.

The experimental procedure for the emergence test consisted of the following steps. A total number of 15 larvae were transferred from their housing tank to a small Petri dish $(3.3 \mathrm{~cm}$ diameter) by means of a $3 \mathrm{ml}$ Pasteur pipette. Thereupon, the content (ca. $4 \mathrm{ml}$ ) of the Petri dish was carefully transferred into the holding compartment, with the passage tube blocked, and the novel environment compartment was filled with the same amount of egg water. After acclimatization of the larvae for $15 \mathrm{~min}$ the passage tube was opened and the larvae could emerge into the other compartment.

The larvae were divided into three groups of five individuals according to the emergence sequence: early, intermediate and late emergers, based on the sequence in which they emerged from the holding compartment. Early emerging (EE) and late emerging (LE) larvae were collected in separate emergence tests to prevent disturbance by removal of larvae during the test. In the EE test the first five larvae were allowed to emerge and were subsequently collected, and in the LE test the first 10 larvae were allowed to emerge, and the remaining larvae were collected. Batches of larvae that took longer than $10 \mathrm{~min}$ to provide the first five EE larvae (which occurred in $<1 \%$ of the batches) were excluded from the experiment (cf. Huntingford et al., 2010). At the end of the emergence test, EE or LE larvae were transferred to separate Petri dishes and left to acclimatize for $20 \mathrm{~min}$, before proceeding with the next assay.

In order to validate our splitting paradigm, a separate emergence test was performed to investigate the distribution of individual emergence times of all 15 larvae in one batch (Figure 2). Ten batches of 15 larvae were allowed to emerge and their emergence times were determined, but without the segregation procedure for sampling the first five or the last five larvae to emerge, as described above.

\section{Open field swimming kinematics before and after exposure to stress}

Between 9 and 11 am, six larvae were transferred to a 12-well plate (Corning Costar, Corning, NY) and placed individually in (different) sustaining nets $(1.5 \mathrm{~cm}$ diameter, water depth $0.7 \mathrm{~cm})$ in separate but adjacent $(2 \times 3)$ wells to ensure a higher optical resolution. The well plate was placed on a light box illuminated by a low-energy LED light (Calex, The Netherlands, 10 Watt, 610 lumen), positioned at $40 \mathrm{~cm}$ under a digital camera (EcoLine, Security Center, Germany, Lens: Cosmicar Pentax TV, 25 mm, 1:1.4). After an acclimatization period of $10 \mathrm{~min}$, the larvae were recorded over a $15 \mathrm{~min}$ period, and swimming kinematics were analyzed using EthoVision XT 6 (Noldus Information Technology b.v., Wageningen, The Netherlands).
Figure 1. Experimental protocol and time line. (A) After splitting individuals in early and late emergers using an emergence test, baseline data were collected for both locomotion activity (behavioral recording in a twelve-well plate) and whole-body cortisol levels. Then, a netting stress paradigm was applied, and locomotion activity and cortisol levels over time were measured after the stress event. (B) Time line of the experiment with durations of the different procedures indicated.
(A) emergence test (ET)

behavioural recording 1 (BR1)

behavioural recording 2 (BR2)

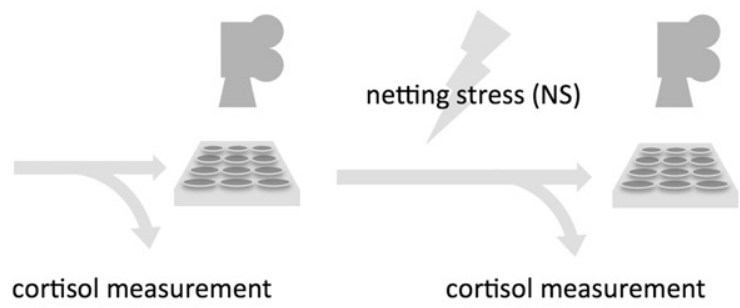

(B)

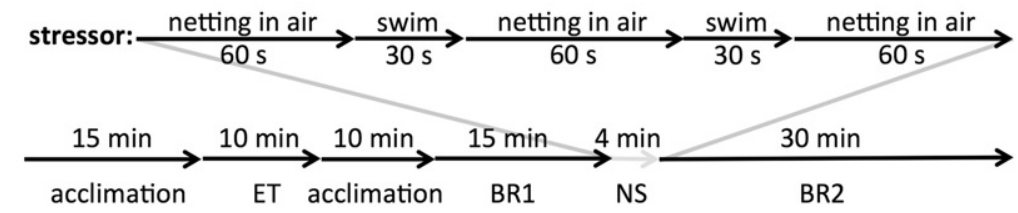




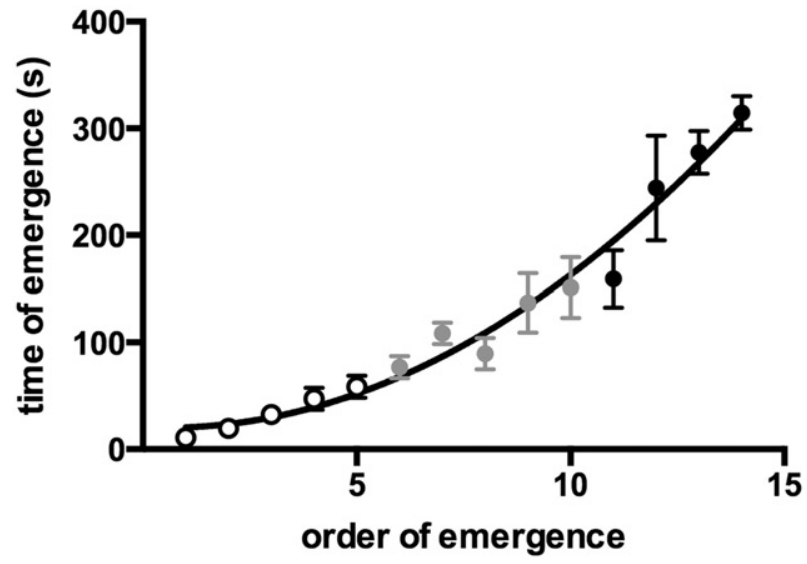

Figure 2. Time of emergence $\left(t_{\mathrm{E}}\right)$ plotted against ranking in the emergence sequence $\left(s_{\mathrm{E}}\right)$ of 14 larvae emerging within 10 min during an emergence test. Ten batches of 15 larvae were allowed to emerge without sampling, and their emergence times were recorded. Data shown are means \pm SEM. The resulting curve was fitted using the function: $t_{\mathrm{E}}=1.5832 s_{E}^{2}-1.7282 s_{\mathrm{E}}+20.467\left(r^{2}=0.74\right)$. Empty and full circles correspond with early and late emergers, respectively, grey circles are intermediate emergers.

Subsequently, a netting stressor was applied by lifting the six sustaining nets out of the water. This way, the six larvae were simultaneously suspended three times in air for $1 \mathrm{~min}$, with two intervals of $30 \mathrm{~s}$ during which they were submerged in the water. Finally, the larvae were released again in their individual well-plate compartments after which we assessed the post-stress swimming kinematics for $30 \mathrm{~min}$.

\section{Analysis of kinematic parameters}

Cumulative distance moved $\left(D_{\text {cum }}\right)$ and swimming velocity $(V)$ were determined using EthoVision XT 6 (Noldus Information Technology b.v.). Turning radii were calculated from $X Y$ coordinate values, according to Domenici \& Blake (1991). The turning radius is the radius of the circle that can be generated using the positions of the centre of mass in three consecutive frames. The sharpness of the turns in a swimming trajectory is best reflected by the minimum turning radius in that trajectory $\left(R_{\min }\right)$, which is here defined as the minimum value of the turning radius within a period of one minute. In general, a higher minimum turning radius indicates straighter swimming paths.

Pre-stress baseline values for $D_{\text {cum }}$ were the values at $t=15 \mathrm{~min}$ and baseline values for $V$ and $R_{\min }$ were the average over a period of $15 \mathrm{~min}$, before the stress event. After the stress event, averages of $1 \mathrm{~min}$ were calculated for $V$ and $R_{\min }$, and a linear regression analysis (GraphPad Prism 6; $\left.y=y_{0}+a t\right)$ resulted in slope $(a)$ and intercept $\left(y_{0}\right)$, which were subsequently used to calculate the recovery time by extrapolation to pre-stress baseline levels $\left(y ; t=\left(y-y_{0}\right) / a\right)$.

\section{Cortisol measurement}

For the assessment of the cortisol response over time after stress, we first used larvae of undetermined coping styles: 10 larvae (in triplicate) were sampled before (baseline) and 5, 10, $15,20,30,60$ and $120 \mathrm{~min}$ after the start of the stressor. Second, EE and LE larvae were exposed to netting stress and samples were taken before (baseline), and 10, 30 and $60 \mathrm{~min}$ after the start of the stressor. For sampling, larvae were snapfrozen in liquid nitrogen and stored at $-20^{\circ} \mathrm{C}$. Whole-body cortisol measurements were carried out using a cortisol ELISA kit (Demeditec) according to the manufacturer's instructions, similar to Tudorache et al. (2013).

\section{Statistics}

Kinematic parameters before the stress event were analyzed using student's $t$-tests (comparison EE and LE fish). $T_{\text {crit }}$ was determined by two-way ANOVA, with time and coping styles (i.e. EE/LE) as the independent variables, and $D_{\text {cum }}$ as the dependent variable. A Sidak's multiple comparison test was used post hoc to determine the exact time point of significant divergence between $D_{\text {cum }}$ values. Two-way ANOVA with Sidak's post-hoc was also used to analyze differences in slopes, before and after $T_{\text {crit, }}$ and between coping styles. Two-way ANOVA with Sidak's post-hoc was finally used to analyze cortisol concentrations, with time and coping styles as the independent variables and cortisol concentrations as the dependent variable. Unless mentioned otherwise, $N$ was 10 and statistical significance was accepted at $p<0.05$. All tests were conducted using SigmaStat 3.0 (Systat Software, Inc., San Jose, CA). All values are presented as mean \pm SEM.

\section{Results}

\section{Emergence test}

For the analysis of emergence behavior, 10 batches of 15 larvae were allowed to emerge (without sampling) for a period of $10 \mathrm{~min}$. The resulting emergence time of each larva was plotted against its ranking in the emergence sequence (Figure 2). The first larva emerged within approximately $20 \mathrm{~s}$ after opening of the passage, the one before last after approximately $5 \mathrm{~min}$. The last larva never emerged into the second compartment within the given time of $10 \mathrm{~min}$. A bestfit analysis of the curve resulted in a second order polynomial function $\left(r^{2}=0.74\right)$. The graph depicts clear differences between the average values for the first five (EE) and the last five (LE) emergers and suggests a homogeneous continuum with larger time differences between subsequent ranks for the late-emergers as compared to the early-emergers.

\section{Open field swimming kinematics before netting stress in EE and LE larvae}

After the emergence test and segregation of EE and LE, open field swimming kinematics of $\mathrm{EE}$ and LE larvae were analyzed. The results showed a higher activity level in LE than in EE larvae. The cumulative distance $\left(D_{\text {cum }}\right)$ travelled by the larvae after $15 \mathrm{~min}$ of swimming activity was significantly lower in EE larvae than in LE larvae $(t(18)=2.191, p<0.05$, Figure 3A). In addition, the average swimming velocity $(V)$ appeared to be approximately three times lower in EE larvae than in LE larvae $(t(18)=6.23$, $p<0.0001$, Figure 3B). Finally, the minimum turning radius $\left(R_{\text {min }}\right)$ was higher in $\mathrm{EE}$ than in LE larvae, indicating straighter swimming paths for the EE larvae $(t(18)=3.25$, $p<0.01$, Figure 3C). 
Figure 3. Kinematic parameters of locomotion behavior in early emerging (empty circles) and late emerging (full circles) larvae before and after netting stress. (A) Cumulative distance moved ( $\left.D_{\text {cum }}\right)$ over $15 \mathrm{~min}$, recorded before netting stress. (B) Swimming velocity $(V)$ averaged over $15 \mathrm{~min}$, recorded before netting stress. (C) Minimum turning radius $\left(R_{\min }, \mathrm{mm}\right)$ average over $15 \mathrm{~min}$, recorded before netting stress. (D) $D_{\text {cum }}$ over time after netting stress ( $1 \mathrm{~min}$ intervals). $T_{\text {crit }}$ indicates the earliest time point $(893 \mathrm{~s})$ at which there is a significant difference between LE and EE values of $D_{\text {cum }}$ values. (E) $V$ over time after netting stress (1 min averages). Lines indicate linear regression curves. (F) $R_{\min }$ over time after netting stress (1 min averages). Lines indicate linear regression curves. All values are average \pm SEM. *Indicates significant differences in values $(\mathrm{A}-\mathrm{C})$ or slopes $(\mathrm{D}-\mathrm{F})$ $(p<0.05)$. before

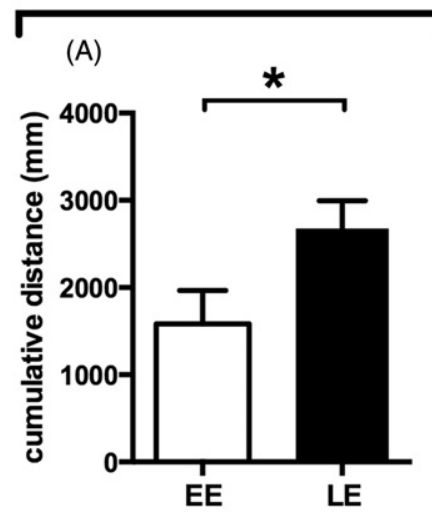

(B)

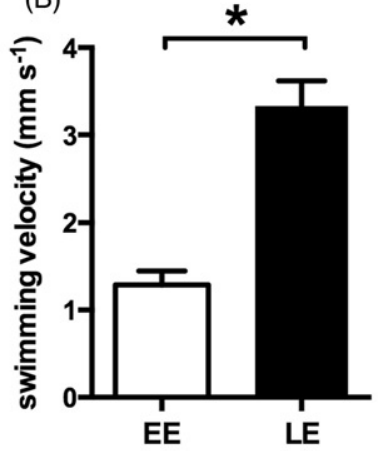

(C)

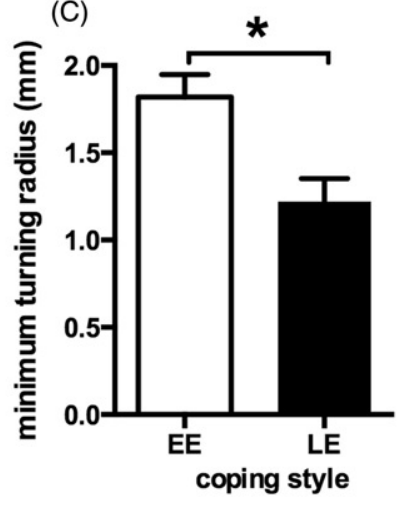

after

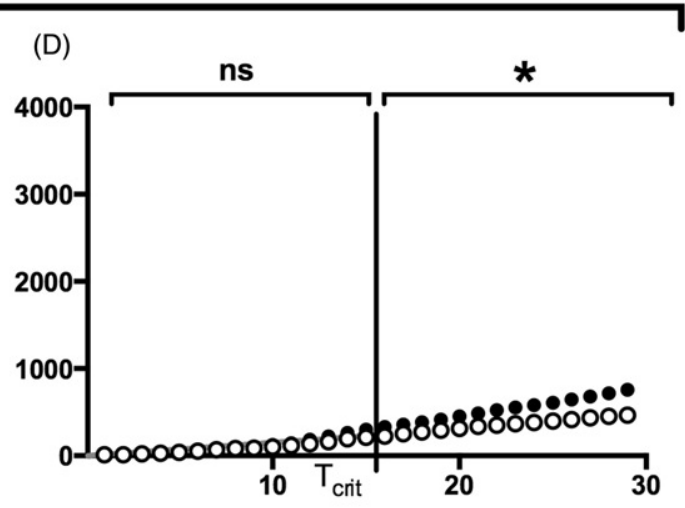

(E)

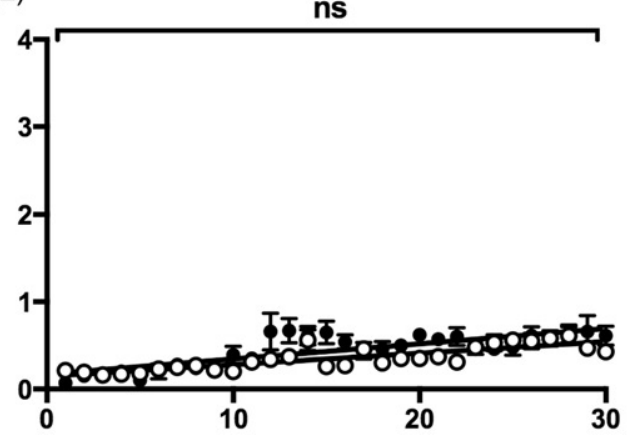

(F)

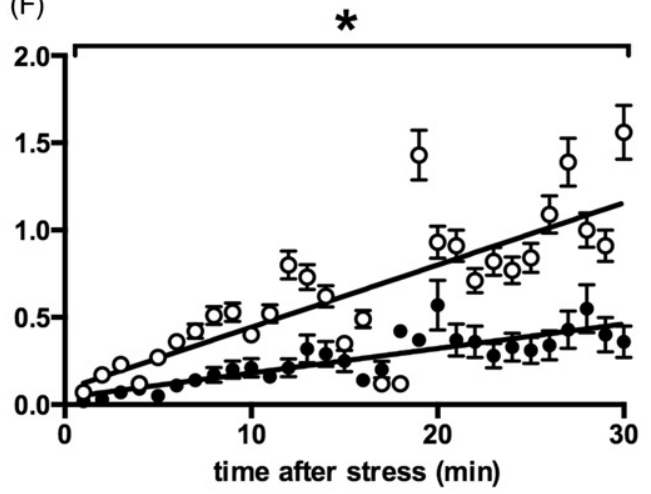

\section{Open field swimming kinematics after netting stress in EE and LE larvae}

EE and LE larvae were exposed to netting stress and subsequent open field swimming kinematics were analyzed for a period of $30 \mathrm{~min}$. $D_{\text {cum }}$ values (Figure 3D) did not differ between EE and LE larvae during the first $893 \mathrm{~s}\left(T_{\text {crit }}\right)$ after netting stress, with both groups showing very low mobility ( $D_{\text {cum }}>10$ times lower than before netting stress). A linear fit of the data over time showed no difference in slope between the EE and LE larvae before $T_{\text {crit }}(0.22 \pm 0.05$ and $0.23 \pm 0.07$ respectively). After $T_{\text {crit }}$, the slopes of the curves became significantly steeper and the slope was significantly lower in $\mathrm{EE}$ than in LE larvae (respectively $0.45 \pm 0.03$ and $0.25 \pm 0.02$; time $F(1,36)=7.184$, coping style $F(1,36)=5.069$, interaction $F(1,36)=4.149, p<0.005$ in all cases). One-minute averages of velocity $(V)$ plotted against time (Figure 3E) showed no difference between EE and LE slopes, before or after $T_{\text {crit }}(0.012 \pm 0.001$ and $0.013 \pm 0.001$ before, and $0.016 \pm 0.002$ and $0.017 \pm 0.002$ after; time $F(1,36)=3.6, \quad p=0.0674$, coping style $F(1,36)=0.22$, $p=0.64$, interaction $F(1,36)=0.0, p>0.99) . R_{\min }$ increased over time in both EE and LE larvae (Figure 3F), reflecting a recovery towards straighter swimming paths. The slope of the linear curve of $R_{\min }$ over time was significantly higher in EE than in LE larvae $(0.034 \pm 0.006$ and $0.013 \pm 0.001$ before, and $0.036 \pm 0.005$ and $0.016 \pm 0.001$ after; time $F(1,36)=0.39, \quad p=0.53, \quad$ coping style $F(1,36)=26.68$, $p<0.001$, interaction $F(1,36)=0.016, p=0.9)$. In summary, the results indicate a lower post-stress activity and straighter swimming trajectories in EE than in LE larvae, similar to the data observed before the netting stress.

\section{Recovery times after netting stress in EE and LE larvae}

In order to relate the values of the kinematic parameters after stress to the basal values, the recovery time was calculated for $V$ and $R_{\min }$. This was done by extrapolating the curves shown 

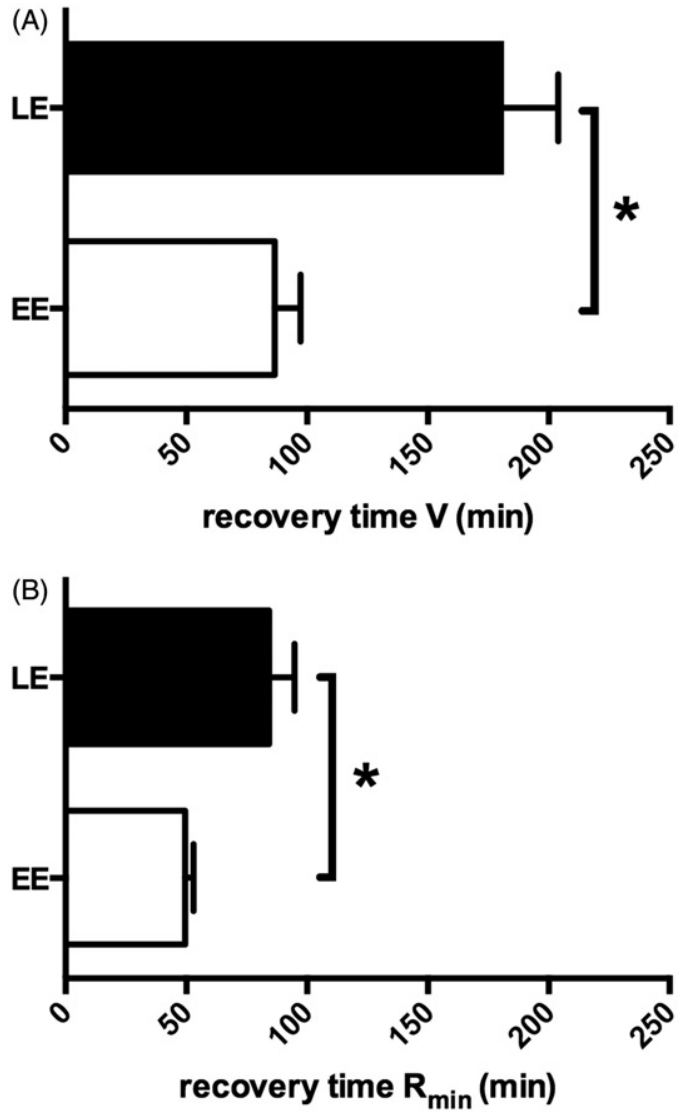

Figure 4. Recovery time to baseline levels as extrapolated from linear regression parameters in early emerging (EE) and late emerging (LE) larvae after netting stress. (A) Recovery time to baseline levels of $V$. (B) Recovery time to baseline levels of $R_{\min }$. All values are mean $\pm \mathrm{SEM}$. *Indicates significant differences $(p<0.05)$.

in Figure 3(E) (EE: $y=(0.013 \pm 0.001) t+(0.14 \pm 0.01)$; LE: $y=(0.017 \pm 0.002) t+(0.16 \pm 0.02))$ and F (EE: $y=(0.035 \pm$ $0.005) t+(0.087 \pm 0.01) ; \quad$ LE: $\quad y=(0.014 \pm 0.001) t+(0.04 \pm$ $0.001)$ ) to baseline values. The recovery time for $V$ was half as long for EE larvae than for LE larvae $(t(18)=3.804$, $p<0.005$, Figure $4 \mathrm{~A}$ ), as was the recovery time for $R_{\min }$, $(t(18)=3.153, p<0.01$, Figure 4B). Thus, the extrapolated recovery of $V$ and $R_{\min }$ to baseline levels is faster in EE than in LE larvae.

\section{Cortisol measurements}

Whole-body cortisol concentrations before (baseline) and after netting stress (peak and recovery) were analyzed. First, the cortisol response was determined for a random batch of larvae of undetermined coping style. The peak moment was determined to be at $10 \mathrm{~min}$ after the onset of the netting stressor, whereas recovery to basal levels was reached at $60 \mathrm{~min}$ after the stressor (Figure 5A). Second, cortisol levels over time were analyzed in EE and LE larvae (Figure 5B; time $F(3,72)=100.5, p<0.001$, coping style $F(1,72)=4.103$, $p<0.05$, interaction $F(3,72)=12.12, \quad p<0.001)$, which revealed a similar baseline cortisol concentration for EE and LE larvae. However, the peak levels were significantly higher in EE than in LE larvae. Additionally, cortisol levels in EE larvae showed a faster recovery than those in LE larvae,
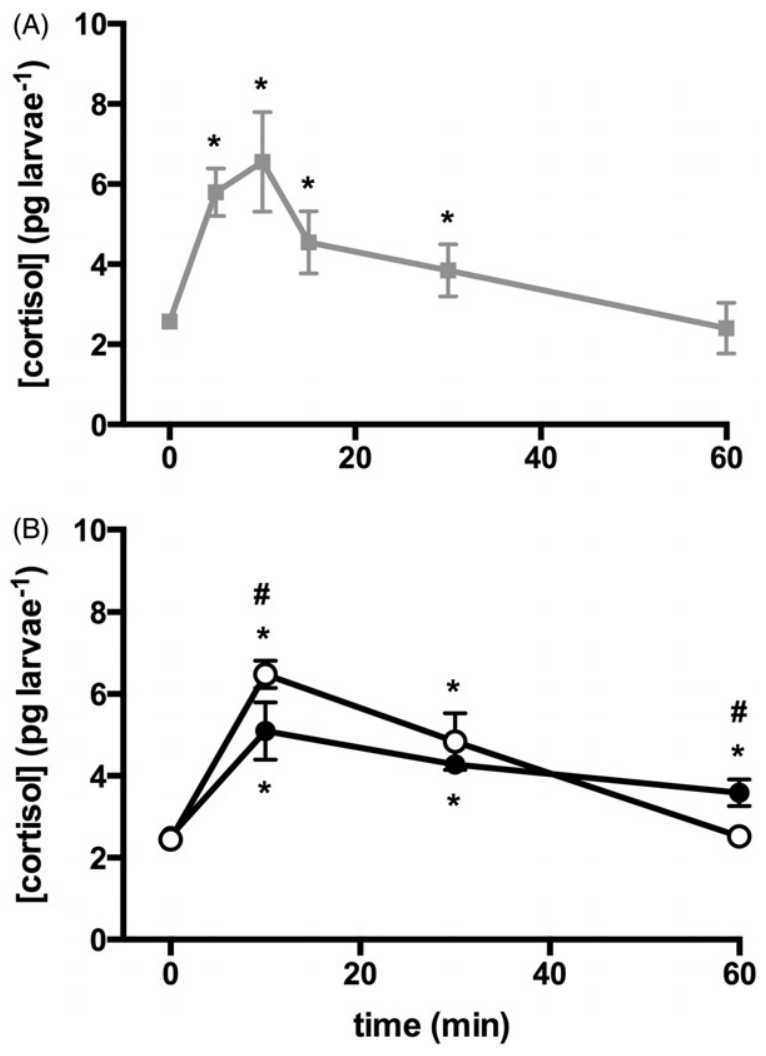

Figure 5. Whole-body cortisol concentration per larva $\left(\mathrm{pg} \mathrm{larva}^{-1}\right)$ after netting stress. (A) Cortisol concentrations at baseline (0) and 5, 10, 15, 30, 60 and $120 \mathrm{~min}$ after the start of the stressor. Larvae were of undetermined coping style. Cortisol levels peak at $10 \mathrm{~min}$ and are back to basal level at $60 \mathrm{~min}$ after the start of the stressor. *Indicates significant difference from baseline levels $(p<0.05, N=10)$. (B) Cortisol concentrations at baseline (0) and 10, 30 and $60 \mathrm{~min}$ after the start of the stressor in different coping styles (EE empty, LE full circles). At the peak of the curve $(10 \mathrm{~min})$, the EE larvae show higher cortisol levels, and reach baseline levels faster than LE larvae. All values are mean \pm SEM. *Indicates significant difference from baseline levels, and \#indicates differences between coping styles $(p<0.05, N=10)$.

reaching baseline levels after $60 \mathrm{~min}$ in $\mathrm{EE}$, while $\mathrm{LE}$ cortisol levels were still elevated at this time point.

\section{Discussion}

The present study demonstrates the association between behavioral and physiological indicators of coping styles in zebrafish larvae. Based on an emergence test, larvae were split into two groups of extreme behavioral phenotypes, i.e. early (EE) and late (LE) emergers. Subsequently, they revealed different locomotion patterns in another behavioral test, and different stressor-induced cortisol response patterns in a physiological test. After the stress event, the recovery rate back to baseline values of both, locomotion patterns and cortisol concentrations, was faster in EE than in LE larvae. Whole-body cortisol levels in EE larvae showed the same baseline levels as LE larvae, but stress-induced peak levels were higher and the recovery rate to baseline levels was faster in EE than in LE larvae.

The current findings for larvae are similar to those in a previous study for adult zebrafish (Tudorache et al., 2013) and consistent with other reports on the early presence of 
individual variation in larvae with respect to behavior (Budaev \& Andrew, 2009) and physiology (Alsop \& Vijayan, 2008). The functional significance of coping styles at an early life stage might be related to selection pressures on particular decisions that vary with environmental conditions (Korte et al., 2005; Reale et al., 2007). It is also increasingly acknowledged that larval fish are not just a passive life stage, but can make active decisions with significant consequences for survival, growth and eventually reproductive opportunities (e.g. Simpson et al., 2005; Stobutzki \& Bellwood, 1997). Zebrafish live and spawn in habitat that is highly variable in time and space (Spence et al., 2008). Consequently, there may well be an ecological explanation of fluctuating selections pressures at the larval stage that explains the presence of coping styles (cf. Bell \& Sih, 2007; Dingemanse \& Reale, 2005). Emergence from a sheltered area can for example be related to emergence tendency from natural spawning nests in nature, as found for larval rainbow trout, Oncorhynchus mykiss (Andersson et al., 2013), which concerns just one decision that may directly affect fitness. We obviously need more ecological studies on larvae in the real world to get a better understanding of the potentially wide-spread occurrence of coping styles very early in development (Höglund et al., 2008; Koprivnikar et al., 2012; McCormick \& Meekan, 2010).

Our methodological strategy for splitting the population into groups bears the advantage of dividing a large number of individuals in a relatively short time into broad but distinct categories of divergent behavioral and endocrine coping strategies. However, it does not allow for correlative analyses based on the full range of the phenotypic continuum and the resolution does not provide insight into the contribution of "rare" phenotypes at the extreme ends of the distribution. Based on our experience with the test procedures in the current study, we believe that individual assessment of coping strategies is feasible for zebrafish larvae (c.f. Budaev \& Andrew, 2009), which will open up yet another avenue of scientific opportunity for the zebrafish model.

We exploited the concept of a widely-used emergence test, modified for the use with young fish larvae, but we refrained from using the coping style labels typically associated with this test: pro-active and re-active (e.g. Huntingford et al., 2010; Huntingford \& Coyle, 2007; MacKenzie et al., 2009). Different from a previous study on adult zebrafish (Tudorache et al., 2013), we here referred to early (EE) and late emergers (LE). The reason for this is that our results show an unexpected negative correlation of emergence sequence and swimming activity, in contrast to the expected positive correlation typical for variation in the behavioral syndrome along the pro-active/re-active axis (c.f. Sih et al., 2004). Therefore we decided to use less interpretative and more descriptive labels.

The negative correlation between emergence time and swimming activity is unexpected, as an overall higher activity of LE larvae should have led to a higher chance to encounter the passage to the novel environment compared to the EE larvae. There are several possible explanations for this apparent inconsistency in the stereotypic dichotomy in coping styles. Light conditions, for example, may also have affected emergence tendency in our setup and variation in scototaxis could alter the link between the explorative nature of individuals and the mobility of individuals. Variation in accommodation time and conditions before testing individual swimming activity may also have affected the results as inherent to the test: there is variation in how long larvae spend in relative darkness before emerging and in whether or not emergence into a novel environment similar to the subsequent test arena was voluntary. Also, social conditions may play a role, as we tested individuals in groups that inherently become smaller over the time course of the test. The fact that the last one of 15 larvae in our validation test never emerged within the given $10 \mathrm{~min}$ of the test, suggests indeed that the social situation may affect stress levels and emergence decisions. In future studies, we aim to investigate the causal mechanism underlying the unexpected negative correlation.

In contrast to the negative correlation between the two different behavioral axes of coping style, we found the expected positive correlation in the recovery to baseline levels for both behavioral and physiological measures, like in our previous study on adult zebrafish (Tudorache et al., 2013): EE recovered faster than LE larvae both in terms of behavior and physiology. After the stress event, locomotion activity was considerably reduced in both EE and LE larvae, in line with previous data in adult zebrafish (Cachat et al., 2010). However, EE larvae subsequently recovered faster to basal locomotion patterns, extrapolated from the calculated recovery times for swimming velocity $(V)$ and minimum turning radius $\left(R_{\min }\right)$. The difference in recovery times corresponds to previous findings in adult zebrafish (Tudorache et al., 2013). This faster recovery in both, behavioral and physiological aspects of coping style after stress, indicates a more dynamic stress coping in EE than in LE larvae, both behaviorally and physiologically. These findings show that recovery times, rather than absolute values, may provide additional insight into behavioral and physiological aspects of coping styles.

The post-stress cortisol levels and recovery dynamics in the current study varied with emergence order in larval zebrafish, similar to previous findings for adult fish (Tudorache et al., 2013) and comparable to findings in mammals. Baseline levels before stress were similar between EE and LE larvae, but EE larvae showed higher peak levels and a faster recovery rate back to baseline levels than LE larvae. Veenema et al. (2003) showed that mice (Mus musculus) with short attack latency (SAL) towards conspecifics had also similar baseline corticosterone levels but faster recovery after stress compared to mice with long attack latency (LAL) This difference between behavioral types was associated with a higher stress-related expression of mineralocorticoid receptors (MR) in the brains of LAL mice, but not of SAL mice, with the MR being linked to fear induced immobility (Korte et al., 1995).

The mechanistic congruence in stress response between fish and mammals is also confirmed by studies on rainbow trout populations genetically selected for divergent post-stress plasma cortisol levels. Johansen et al. (2011) showed that low responsive (LR) fish have a higher MR expression than high responsive (HR) fish. The LR fish also had a reduced locomotor response in a stress test compared to HR fish (Øverli et al., 2002), which is similar again to the lower poststress activity of the EE larvae in our study. The relationship 
between HPI-axis expression and behavioral syndromes is complex (Koolhaas et al., 2010), but our findings indicate a more dynamic cortisol response to stress in EE larvae, possibly based on differences in feedback mechanism of the HPI-axis. The comparison among studies on different taxa reveals the congruence of stress coping styles across vertebrate species (c.f. Steenbergen et al., 2011).

\section{Conclusion}

The present study shows that different coping styles are present in zebrafish larvae at $8 \mathrm{dpf}$, and that behavioral and physiological characteristics of coping styles are correlated. Furthermore, the data on swimming kinematics indicate clearly different movement patterns before and after acute netting stress and strong differences in these patterns between coping styles. Finally, the recovery rate after stress is consistently faster in early than in late emerging larvae, for both behavioral (swimming velocity and movement pattern) and physiological parameters (cortisol level). These data provide new insights into individual variation very early in development of the endocrine and behavioral stress response for a typical vertebrate species and confirms that zebrafish larvae are a useful model for studies that may yield an important contribution to medical applications, animal welfare issues and conservation.

\section{Acknowledgements}

We would like to acknowledge the technical support of Davy de Witt and the helpful discussions with Herman Spaink and Peter Steenbergen.

\section{Declaration of interest}

There is no conflict of interest that could be perceived as prejudicing the impartiality of the research reported. The authors alone are responsible for the content and writing of the paper.

CT was supported by an NWO-STW funded VENIfellowship (\#11731).

\section{References}

Alsop D, Vijayan MM. (2008). Development of the corticostreroid stress axis and receptor expression in zebrafish. Am J Physiol Regul Integr Comp Physiol 294:R711-19.

Andersson MA, Khan UW, Øverli Ø, Gjøn HM, Höglund, E. (2013). Coupling between stress coping style and time of emergence from spawning nests in salmonid fishes: evidence from selected rainbow trout strains (Oncorhynchus mykiss). Physiol Behav 116-117:30-4.

Bell AM, Sih A. (2007). Exposure to predation generates personality in threespined sticklebacks (Gasterosteus aculeatus). Ecol Lett 10: 828-34.

Bernier NJ, Bedard N, Peter RE. (2004). Effects of cortisol on food intake, growth, and forebrain neuropeptide $\mathrm{Y}$ and corticotropinreleasing factor gene expression in goldfish. Gen Comp Endocrinol 135:230-40.

Budaev S, Andrew R. (2009). Shyness and behavioural asymmetries in larval zebrafish (Brachydanio rerio) developed in light and dark. Behaviour 146:1037-52.

Cachat J, Stewart A, Grossman L, Gaikwad S, Kadri F, Chung KM, Wu $\mathrm{N}$, et al. (2010). Measuring behavioral and endocrine responses to novelty stress in adult zebrafish. Nat Prot 5(11):1786-99.

Champagne DL, Hoefnagels CCM, De Kloet RE, Richardson MK. (2010). Translating rodent behavioral repertoire to zebrafish
(Danio rerio): relevance for stress research. Behav Brain Res 214: 332-42.

Coleman K, Wilson DS. (1998). Shyness and boldness in pumpkinseed sunfish: individual differences are context-specific. Anim Behav 56: 927-36.

Conrad JL, Weinersmith KL, Brodin T, Saltz JB, Sih A. (2011). Behavioural syndromes in fishes: a review with implications for ecology and fisheries management. J Fish Biol 78:395-435.

Consten D, Lambert JGD, Komen H, Goos HJT. (2002). Corticosteroids affect the testicular androgen production in male common carp (Cyprinus carpio L.). Biol Repr 66:106-11.

De Kloet ER, Vreugdenhil E, Oitzl MS, Joels M. (1998). Brain corticosteroid receptor balance in health and disease. Endocrine Rev 19(3):269-301.

Dingemanse NJ, Reale D. (2005). Natural selection and animal personality. Behaviour 142:1159-84.

Domenici P, Blake RW. (1991). The kinematics and performance of the escape response in the angelfish (Pterophyllum eimekei). J Exp Biol 156:187-205.

Egan RJ, Bergner CL, Hart PC, Cachat JM, Canavello PR, Elegante MF, Elkhayat SI, et al. (2009). Understanding behavioral and physiological phenotypes of stress and anxiety in zebrafish. Beh Brain Res 205: $38-44$.

Höglund E, Gjøn HM, Pottinger TG, Øverli Ø. (2008). Parental stresscoping styles affect the behaviour of rainbow trout Oncorhynchus mykiss at early developmental stages. J Fish Biol 73:1764-9.

Huntingford FA. (1976). Relationship between anti-predator behavior and aggression among conspecifics in 3-spined stickleback, Gasterosteus aculeatus. Anim Behav 24:245-60.

Huntingford FA, Andrew G, Mackenzie S, Morera D, Coyle SM, Pilarczyk M, Kadri S. (2010). Coping strategies in a strongly schooling fish, the common carp Cyprinus carpio. J Fish Biol 76(7):1576-91

Huntingford FA, Coyle SM. (2007). Anti-predator defences in sticklebacks: trade offs, risk sensitivity and behavioural syndromes. In: Ostlund-Nilsson S, Mayer I, Huntingford FA, editors. Biology of the three-spined stickleback. Boca Raton, FL: CRC Press. p 127-56.

Johansen IB, Sandvik GK, Nilsson GE, Bakken M, Øverli Ø. (2011). Cortisol receptor expression differs in the brains of rainbow trout selected for divergent cortisol responses. Comp Biochem Physiol D 6: 126-32.

Koolhaas JM, Korte SM, De Boer SF, Van Der Vegt BJ, Van Reenen CG, Hopster H, De Jong IC, et al. (1999). Coping styles in animals: current status in behavior and stress-physiology. Neurosci Biobehav Rev 23(7):925-35.

Koolhaas JM, de Boer SF, Coppens CM, Buwalda B. (2010). Neuroendocrinology of coping styles: towards understanding the biology of individual variation. Front Neuroendocrinol 31:307-21.

Koprivnikar J, Gibson CH, Redfern JC. (2012). Infectious personalities: behavioural syndromes and disease risk in larval amphibians. Proc Roy Soc B 279:1544-50.

Korte SM, De Boer SF, De Kloet ER, Bohus B. (1995). Anxiolytic-like effects of selective mineralocorticoid and glucocorticoid antagonists on fear-enhanced behavior in the elevated plus-maze. Psychoneuroendocrinology 20:385-94.

Korte M, Koolhaas JM, Wingfield JC, McEwen BJ. (2005). The Darwinian concept of stress: benefits of allostasis and costs of allostatic load and the trade-offs in health and disease. Neurosci Biobehav Rev 29:3-38.

MacKenzie S, Ribas L, Pilarczyk M, Capdevila DM, Kadri S, Huntingford FA. (2009). Screening for coping style increases the power of gene expression studies. PLoS One 4(4):e5314.

McCormick MI, Meekan MG. (2010). The importance of attitude: the influence of behaviour on survival at an ontogenetic boundary. Mar Ecol Prog Ser 407:173-85.

Oswald ME, Drew RE, Racine M, Murdoch GK, Robison BD. (2012). Is behavioral variation along the bold-shy continuum associated with variation in the stress axis in zebrafish? Physiol Biochem Zool 85: 718-28.

Øverli Ø, Kotzian S, Winberg S. (2002). Effects of cortisol on aggression and locomotor activity in rainbow trout. Horm Behav 42:53-61.

Øverli Ø, Sorensen C, Pulman KGT, Pottinger TG, Korzan WJ, Summers CH, Nilsson GE. (2007). Evolutionary background for stress-coping styles: relationships between physiological, behavioral, and cognitive 
traits in non-mammalian vertebrates. Neurosci Biobehav Rev 31(3): 396-412.

Ramsay JM, Feist GW, Varga ZM, Westerfield M, Kent ML, Schreck CB. (2006). Whole-body cortisol is an indicator of crowding stress in adult zebrafish, Danio rerio. Aquaculture 258: 565-74.

Raoult V, Brown C, Zuberi A, Williamson JE. (2012). Blood cortisol concentrations predict boldness in juvenile mulloway (Argyosomus japonicus). J Ethol 30(2):225-32.

Reale D, Reader SM, Sol D, McDougall PT, Dingemanse NJ. (2007). Integrating animal temperament within ecology and evolution. Biol Rev 82:291-318.

Schjolden J, Stoskhus A, Winberg S. (2005). Does individual variation in stress responses and agonistic behavior reflect divergent stress coping strategies in juvenile rainbow trout? Physiol Biochem Zool 78(5): $715-23$.

Schreck CB, Contreras-Sanchez W, Fitzpatrick MS. (2001). Effects of stress on fish reproduction, gamete quality, and progeny. Aquaculture 197:3-24.

Sih A, Bell A, Johnson JC. (2004). Behavioral syndromes: an ecological and evolutionary overview. Trends Ecol Evol 19:372-8.
Simpson SD, Meekan M, Montgomery J, McCauley R, Jeffs A. (2005). Homeward sound. Science 308:221.

Spence R, Gerlach G, Lawrence C, Smith C. (2008). The behaviour and ecology of the zebrafish, Danio rerio. Biol Rev 83:13-34.

Steenbergen PJ, Richardson MK, Champagne DL. (2011). The use of the zebrafish model in stress research. Progr Neuro-Psychopharmacol Biol Psychiatry 35:1432-51.

Stobutzki IC, Bellwood DR. (1997). Sustained swimming abilities of the late pelagic stages of coral reef fishes. Mar Ecol Prog Ser 149:35.

Tudorache C, Schaaf MJM, Slabbekoorn H. (2013). Covariation between behaviour and physiology indicators of coping style in zebrafish (Danio rerio). J Endocrin 219(3):251-8.

Veenema AH, Meijer OC, De Kloet ER, Koolhaas JM, Bohusa BG. (2003). Differences in basal and stress-induced HPA regulation of wild house mice selected for high and low aggression. Horm Behav 43:197-204.

Vignet C, Begout ML, Pean S, Lyphout L, Leguay D, Cousin X. (2013). Systematic screening of behavioral responses in two zebrafish strains. Zebrafish 10(3):365-75.

Wendelaar-Bonga SE. (1997). The stress response in fish. Physiol Rev 77(3):591-625. 